\title{
An Iterative Algorithm for Robust Kernel Principal Component Analysis
}

\author{
Hsin-Hsiung Huang ${ }^{\mathrm{a}}$, Yi-Ren Yeh $^{\mathrm{b}}$ \\ ${ }^{a}$ Department of Mathematics, Statistics and Computer Science of University of Illinois at Chicago, Chicago, IL, US \\ ${ }^{b}$ Research Center for Information Technology Innovation, Academia Sinica, Taipei, Taiwan
}

\begin{abstract}
We introduce a technique to improve iterative kernel principal component analysis (KPCA) robust to outliers due to undesirable artifacts such as noises, alignment errors, or occlusion. The proposed iterative robust KPCA (rKPCA) links the iterative updating and robust estimation of principal directions. It inherits good properties from these two ideas for reducing the time complexity, space complexity, and the influence of these outliers on estimating the principal directions. In the asymptotic stability analysis, we also show that our iterative rKPCA converges to the weighted kernel principal kernel components from the batch rKPCA. Experimental results are presented to confirm that our iterative rKPCA achieves the robustness as well as time saving better than batch KPCA.
\end{abstract}

Keywords:

Kernel principal component analysis, iterative update, outliers, robust estimation.

\section{Introduction}

Principal component analysis (PCA) is a classical dimension reduction method which has been applied in many applications, such as data visualization, image reconstruction, biomedical study, etc. It is a linear transformation that searches for an orthonormal basis of a low-dimensional subspace, so called the principal components subspace, which explains the variability of the data as much as possible [1]. PCA's utility and success stem from the simplicity of the method that calculates the eigenvectors and eigenvalues of the sample covariance matrix of the data set.

While in some cases, a linear transformation is not suitable for capturing the nonlinear structures of the data, in order to represent the nonlinear structure, the kernel PCA (KPCA) has been formulated [2] in a reproducing kernel Hilbert space framework. In KPCA, the computational cost depends on the sample size. When the sample size is very large, it is impractical to compute the principal components via a direct eigenvalue

Email addresses: hhuang45@uic . edu (Hsin-Hsiung Huang), yryeh@citi.sinica.edu.tw (Yi-Ren Yeh) decomposition. Given a matrix $X=\left[x_{1}, x_{2}, \cdots, x_{m}\right] \in$ $\mathbb{R}^{n \times m}$ of $m$ observations in an $n$-dimensional space, the batch KPCA through direct eigenvalue decomposition on the covariance of the kernel matrix $K \in \mathbb{R}^{m \times m}$ needs $O\left(\mathrm{~m}^{2}\right)$ space and $O\left(\mathrm{~m}^{3}\right)$ computation time, where $K_{i j}=K\left(x_{i}, x_{j}\right)$ is a kernel function $K(x, u)$. In contrast, given $l$ principal components, the iterative KPCA costs $O\left(m\left(n+l^{2}\right)\right)$ time complexity and $O(m(n+l))$ space in each updating iteration [3].

Furthermore, the conventional PCA is quite sensitive to outliers. Several approaches to achieve robustness have been proposed in the literature $[4,5]$. It is also recognized that KPCA is sensitive to outliers, and the analysis based on the contaminated principal components can be misleading [6]. A robust mechanism to reduce the influence of outliers is introduced [6]. In taking the advantages of iterative updating and outliers resistance, in this article we aim for an iterative robust KPCA (rKPCA) algorithm, which updates the principal directions via each kernel datum and automatically assigns a weight to this datum for the robust estimation at the same time. We will also study its theoretical properties as well as plan for real data applications. 
It is also worth noting that there are two relevant work which are similar to ours [7, 8]. Compared to their work, our proposed rKPCA not only advances iterative updating techniques for robust estimation of principal directions, our work studies the asymptotic stability analysis for the iterative rKPCA and proves the convergence. Using any monotonically increasing weight functions, our method confirms the asymptotic convergence of the iterative rKPCA. This asymptotic stability analysis is a critical issue while an iterative updating scheme is used (like iterative KPCA). In the works of [7, 8], only a particular weight function was applied (which is one of the functions we consider and is also used in [5]), and no theoretical backgrounds or convergence guarantee is provided. Therefore, our work is technically more complete than theirs.

The following is the organization of this paper. In Section 2, we review the rKPCA based on a robust psiweight function and the iterative KPCA which is the same as the kernel Hebbian algorithm (KHA) [9]. Then in Section 3, how to adapt the estimation of rKPCA using gradient descent is presented. We show that the mean and the principal components due to the iterative rKPCA are asymptotically stable. The asymptotic behavior of the eigenvectors of sample covariance matrices based on the gradient descent update is summarized in the same section as well. In Section 4, natural and artificial experiments are designed to confirm that the iterative rKPCA can reduce the influence of outliers of data. Conclusive remarks are in Section 5. In the appendix, mathematical proofs confirm the sufficient conditions of the asymptotic properties for the mean and kernel principal components obtained by the iterative rKPCA.

\section{Related Previous Work}

\subsection{Robust Kernel Principal Component Analysis}

Given a matrix $X \in \mathbb{R}^{n \times m}$ of observational data and a positive definite kernel function $K(x, u)$, the associated separable Hilbert space consists of the closure of all finite kernel mixtures $\sum_{i=1}^{\tilde{n}} a_{i} K\left(x, u_{i}\right)$, where $\tilde{n} \in \mathbb{N}, u_{i} \in$ $\mathbb{R}^{n}, a_{i} \in \mathbb{R}$. The norm $\|v\|_{\mathcal{H}}=\left(\langle v, v\rangle_{\mathcal{H}}\right)^{1 / 2}$ is induced by the inner product: $\langle K(x, \cdot), K(u, \cdot)\rangle_{\mathcal{H}}=K(x, u)$. This Hilbert space is known as an RKHS denoted by $\mathcal{H}$.

Assumed that $\mathcal{H}$ is separable and hence has a countable orthonormal (o.n.) basis set, denoted by $\left\{e_{i}\right\}_{i=1}^{\infty}$. Particularly, one may take $e_{i}=\sqrt{\pi_{i}} \phi_{i}$, where $\pi_{i}$ 's and $\phi_{i}$ 's are the eigenvalues and the corresponding eigenfunctions of $K(x, u)$, and then the spectrum decomposition is $K(x, u)=\sum_{i} \pi_{i} \phi_{i}(x) \phi_{i}(u)$ with $\sum_{i} \pi_{i}<\infty$. Each input element $x$ is mapped into an element $h_{x}=K(x, \cdot)$.
Besides, each element $h \in \mathcal{H}$ can be expanded in terms of this o.n. system as

$$
h=\sum_{i} a_{i} e_{i}, \text { where } a_{i}=\left\langle h, e_{i}\right\rangle_{\mathcal{H}} .
$$

Let $O_{l}$ be the collection of all rank- $l$ linear operators of the form $\Gamma=\sum_{i=1}^{l} \gamma_{i} \otimes e_{i}$ with $\left\{\gamma_{i}\right\}_{i=1}^{l}$ being orthonormal in $\mathcal{H}$ where $\otimes$ denotes tensor product. Its transpose can be expressed as $\Gamma^{\top}=\sum_{i=1}^{l} e_{i} \otimes \gamma_{i}$. For $\Gamma \in O_{l}$, let $\mathcal{P}_{\Gamma}$ and $\mathcal{P}_{\Gamma^{\perp}}$ denote, respectively, the orthogonal projections onto the subspace $\operatorname{Im}(\Gamma)=\operatorname{span}\left\{\gamma_{1}, \ldots, \gamma_{1}\right\}$ and its orthogonal complement $\operatorname{Im}\left(\Gamma^{\perp}\right)$. For $\mu \in \mathcal{H}$ and $\Gamma \in O_{l}$, define

$$
\begin{aligned}
z\left(h_{x}, \mu, \Gamma\right) & =\frac{1}{2}\left\{\left\|h_{x}-\mu\right\|_{\mathcal{H}}^{2}-\left\|\Gamma^{\top}\left(h_{x}-\mu\right)\right\|_{\mathcal{H}}^{2}\right\} \\
& =\frac{1}{2}\left\|\mathcal{P}_{\Gamma^{\perp}}\left(h_{x}-\mu\right)\right\|_{\mathcal{H}^{\prime}}^{2} .
\end{aligned}
$$

Note that $z\left(h_{x}, \mu, \Gamma\right)$ is the squared residual norm by fitting $\left(h_{x}-\mu\right)$ to the subspace $\operatorname{Im}(\Gamma)$. The KPCA of leading $l$ principal components looks for $\Gamma \in O_{l}$ that satisfies the smallest expected residual norm. Assumed the data belong to distribution $G$, the KPCA solves the following minimization problem:

$$
\underset{\mu \in \mathcal{H}, \Gamma \in O_{l}, \Gamma^{\top} \Gamma=I}{\operatorname{argmin}} \mathrm{E}_{G} z\left(h_{x}, \mu, \Gamma\right),
$$

where $h_{x}=K(x, \cdot)$ and $x \sim G$. Therefore the minimization problem for samples from a population belonging to distribution $G$ is of the following form

$$
\underset{\mu \in \mathcal{H}, \Gamma \in O_{l}, \Gamma^{\top} \Gamma=I}{\operatorname{argmin}} \sum_{t=1}^{m} z\left(h_{t}, \mu, \Gamma\right)
$$

where $h_{t}=K\left(x_{t}, \cdot\right)$ and $x_{t}$ is iid from $G$. It can be shown that problems (3) and (4) lead to the solutions $(\mu, \Gamma)$, respectively, as

$$
\mu=\mathrm{E}_{G} h_{x} \text { and } \Gamma=\operatorname{eigen}_{l}(\Sigma),
$$

where $\Sigma=\mathrm{E}_{G}\left\{\left(h_{x}-\mu\right) \otimes\left(h_{x}-\mu\right)\right\}$;

$$
\hat{\mu}=\frac{1}{m} \sum_{t=1}^{m} h_{t} \text { and } \hat{\Gamma}=\operatorname{eigen}_{l}(\hat{\Sigma}),
$$

where $\hat{\Sigma}=\frac{1}{m} \sum_{t=1}^{m}\left(h_{t}-\hat{\mu}\right) \otimes\left(h_{t}-\hat{\mu}\right)$ and $\operatorname{eigen}_{l}(M)$ stands for the leading $l$ eigenfunctions of $M$, denoted by $\gamma_{i}$, collected together to form the rank- $l$ operator $\sum_{i=1}^{l} \gamma_{i} \otimes$ $e_{i} \in O_{l}$. In other words, the KPCA solves for the leading eigenfunctions for a covariance operator $\Sigma$ or $\hat{\Sigma}$.

However, the conventional KCPA formulation (4) is not robust [6]. For example, if data are polluted with 
intra-sample outliers, the estimated principal directions are affected by these outliers. As a result, principal components may not lead to greatest variances of unpolluted data. For mitigation of the influence of outliers, Huang et al. [6] proposed a robust version of iterative KPCA based on the the principle of robust loss function that enables the incorporation of robustness and is taken as a constrained minimization problem

$$
\underset{\mu, \Gamma, \Gamma^{\top} \Gamma=I}{\operatorname{argmin}} \sum_{t=1}^{m} \Psi\left(z\left(h_{t}, \mu, \Gamma\right)\right),
$$

where $\Psi(z)$ is a monotonic increasing function of $z$. As the theorem in [6] stated, the minimizer of the above problem will satisfy the following stationary equations:

$$
\begin{gathered}
\mu=\frac{\mathrm{E}_{G}\left[\dot{\Psi}\left(z\left(h_{x}, \mu, \Gamma\right)\right) h_{x}\right]}{\mathrm{E}_{G}\left[\dot{\Psi}\left(z\left(h_{x}, \mu, \Gamma\right)\right)\right]}, \\
\Gamma=\frac{\mathrm{E}_{G}\left[\dot{\Psi}\left(z\left(h_{x}, \mu, \Gamma\right)\right)\left(h_{x}-\mu\right) \otimes\left(h_{x}-\mu\right)\right]}{\mathrm{E}_{G}\left[\dot{\Psi}\left(z\left(h_{x}, \mu, \Gamma\right)\right)\right]},
\end{gathered}
$$

where $\dot{\Psi}(z)=\frac{d \Psi(z)}{d z}$. It will result in assigning smaller weights to the outliers so that the influence of these outliers reduces. Here are a few choices of $\Psi(z)$ and corresponding unnormalized weight function $\dot{\Psi}(z)$ :

- $\Psi_{0}(z)=z$ with $\dot{\Psi}_{0}(z)=1$, which leads to the conventional KPCA.

- $\Psi_{1}(z)=\left(1-e^{-\beta z}\right) / \beta$ with $\dot{\Psi}_{1}(z)=e^{-\beta z}$.

- $\Psi_{2}(z)=-\frac{1}{\beta} \log \left\{\frac{1+e^{-\beta(z-\xi)}}{2}\right\}$ with $\dot{\Psi}_{2}(z)=1-\frac{1}{1+e^{-\beta(z-\xi)}}$.

\subsection{Iterative Kernel Principal Component Analysis}

We apply a gradient descent method to solve the minimization problem (4) iteratively which implements in the same way as the kernel Hebbian algorithm (KHA) [9]. The KHA applies the generalized Hebbian algorithm [10] to the kernel expansion of data and considers the following minimization problem to update the current $\mu_{t}$ and $\Gamma_{t}$ via each arriving kernel datum $h_{t}$ :

$$
\underset{\mu_{t}, \Gamma_{t}, \Gamma_{t}^{\top} \Gamma_{t}=I}{\operatorname{argmin}} z\left(h_{t}, \mu_{t}, \Gamma_{t}\right) .
$$

By introducing the Lagrange multipliers $\Lambda$, the Lagrangian function of (10) can be expressed as follows:

$$
\begin{aligned}
\mathcal{L}\left(\mu_{t}, \Gamma_{t}, \Lambda_{t}\right)= & z\left(h_{t}, \mu_{t}, \Gamma_{t}\right)+\sum_{i=1}^{l} \frac{1}{2} \lambda_{t_{i i}}\left(\left\|\gamma_{t_{i}}\right\|^{2}-1\right) \\
& +\sum_{i>j}^{l} \lambda_{t_{i j}}\left\langle\gamma_{t_{i}}, \gamma_{t_{j}}\right\rangle_{\mathcal{H}} .
\end{aligned}
$$

where $\gamma_{i}(t)$ 's are columns of $\Gamma$ and $\Lambda_{t_{i j}}=\lambda_{t_{i j}}$. Taking the derivatives of (11) with respect to $\mu_{t}$ and $\Gamma_{t}$, we have the gradients of $\mathcal{L}\left(\mu_{t}, \Gamma_{t}, \Lambda_{t}\right)$ with respect to $\mu_{t}$ and $\Gamma_{t}$ as follows:

$$
\begin{aligned}
& g_{t}^{(\mu)}=-\left(h_{t}-\mu_{t}\right), \\
& g_{t}^{(\Gamma)}=-\left(\left(h_{t}-\mu_{t}\right) \otimes\left(h_{t}-\mu_{t}\right)\right) \Gamma_{t}-\Gamma_{t} \mathcal{U} \mathcal{T}\left(\Lambda_{t}\right),
\end{aligned}
$$

where $\mathcal{U T}(\cdot)$ is the operator that picks the upper triangular entries of a matrix and Lagrange multipliers $\Lambda_{t} \in \mathbb{R}^{l \times l}$ are updated as follows

$$
\left\{\begin{array}{l}
\lambda_{t_{i i}}=\gamma_{t_{i}}^{\top}\left(h_{t}-\mu_{t}\right)\left(h_{t}-\mu_{t}\right)^{\top} \gamma_{t_{i}}, \\
\lambda_{t_{i j}}=\gamma_{t_{i}}^{\top}\left(h_{t}-\mu_{t}\right)\left(h_{t}-\mu_{t}\right)^{\top} \gamma_{t_{j}} .
\end{array}\right.
$$

These lead to a iterative updating for $\mu \in \mathbb{R}^{m \times 1}$

$$
\mu_{t+1}=\mu_{t}-\eta_{t} g_{t}^{(\mu)},
$$

and for $\Gamma \in \mathbb{R}^{m \times l}$

$$
\Gamma_{t+1}=\Gamma_{t}-\eta_{t} g_{t}^{(\Gamma)},
$$

where $\eta_{t}=\frac{1}{t}$ is a learning rate at the $t^{\text {th }}$ iteration.

Note that the update process of the principal components is similar to the KHA. However there are some differences. First, we update the mean in (12), but KHA uses an empirical mean which is fixed through the learning process. When sample size is very large, it is not plausible to calculate an empirical mean with the whole data set. In this situation, the adaptive update rule of the mean still can be conducted.

\section{Iterative Robust KPCA and Its Asymptotic Sta- bility}

As mentioned in the previous section, it is impractical to compute the principal components via a direct eigenvalue decomposition while we have a large sample size in KPCA. Besides, it is also recognized that KPCA is sensitive to outliers. To overcome these two problems, we propose an iterative KPCA method, which advances iterative updating techniques for robust estimation of principal directions. We note that, although the number of training instances determines the dimensionality of the kernel in KPCA, an incremental (or online) setting cannot guarantee asymptotic convergence of the iterative KPCA. In our proposed iterative rKPCA, we assume that there are only finite main features of these observations in. That is, we can choose an arbitrary fixed basis of the kernel so that the size of the kernel is fixed as in KHA [9]. Thus the main issue is to find a way to choose the number of principal components to compress the data size, and to prove convergence of our proposed method. 


\subsection{Formulation of Iterative Robust KPCA}

For linking the iterative updating and robust estimation of kernel principal directions, we consider the following single observation version of (7):

$$
\underset{\mu_{t}, \Gamma_{t}, \Gamma_{t}^{\top} \Gamma_{t}=I}{\operatorname{argmin}} \Psi\left(z\left(h_{t}, \mu_{t}, \Gamma_{t}\right)\right) .
$$

Now we introduce Lagrange multipliers for the minimization problem subject to the orthonormal basis constraints. Then we have the Lagrangian function of (16):

$$
\begin{aligned}
\mathcal{L}\left(\mu_{t}, \Gamma_{t}, \Lambda_{t}\right)= & \left.\Psi\left(z\left(h_{t}, \mu_{t}, \Gamma_{t}\right)\right)+\sum_{i=1}^{l} \frac{1}{2} \lambda_{t_{i i}}\left\|\gamma_{t_{i}}\right\|^{2}-1\right) \\
& +\sum_{i>j}^{l} \lambda_{t_{i j}}\left\langle\gamma_{t_{i}}, \gamma_{t_{j}}\right\rangle_{\mathcal{H}} .
\end{aligned}
$$

The gradients of $\mathcal{L}\left(\mu_{t}, \Gamma_{t}, \Lambda_{t}\right)$ with respect to $\mu$ and $\Gamma$ of (17) while receiving a kernel observation $h_{t}$ are

$$
\begin{aligned}
g_{t}^{(\mu)}= & -\dot{\Psi}\left(z\left(h_{t} ; \mu_{t}, \Gamma_{t}\right)\right)\left(h_{t}-\mu_{t}\right), \\
g_{t}^{(\Gamma)}= & -\dot{\Psi}\left(z\left(h_{t} ; \mu_{t}, \Gamma_{t}\right)\right)\left\{\left[\left(h_{t}-\mu_{t}\right) \otimes\left(h_{t}-\mu_{t}\right)\right] \Gamma_{t}\right. \\
& \left.-\Gamma_{t} \mathcal{U} \mathcal{T}\left(\Lambda_{t}\right)\right\},
\end{aligned}
$$

where $\dot{\Psi}(z)$ gives a smaller weight to reduce the effect of misleading updating from an outlier. Here the Lagrange multiplier $\lambda_{t_{i j}}$ in $\Lambda_{t}$ is calculated by using the following Property 1.

Property 1. In the solutions, $\lambda_{t_{i j}}$ 's take the following values:

$\lambda_{t_{i j}}= \begin{cases}\dot{\Psi}\left(z\left(h_{t}, \mu_{t}, \Gamma_{t}\right)\right) \gamma_{t_{t_{i}}}^{\top}\left(h_{t}-\mu_{t}\right)\left(h_{t}-\mu_{t}\right)^{\top} \gamma_{t_{i}}, & i=j, \\ \dot{\Psi}\left(z\left(h_{t}, \mu_{t}, \Gamma_{t}\right)\right) \gamma_{t_{i}}^{\top}\left(h_{t}-\mu_{t}\right)\left(h_{t}-\mu_{t}\right)^{\top} \gamma_{t_{j}} & i<j .\end{cases}$

With the gradient $g_{t}^{(\mu)}$ and $g_{t}^{(\Gamma)}$, the updating for $\mu \epsilon$ $\mathbb{R}^{\tilde{m} \times 1}$ in iterative rKPCA

$$
\mu_{t+1}=\mu_{t}-\eta_{t} g_{t}^{(\mu)}
$$

and for $\Gamma \in \mathbb{R}^{\tilde{m} \times l}$

$$
\Gamma_{t+1}=\Gamma_{t}-\eta_{t} g_{t}^{(\Gamma)}
$$

where the details of the entire updating procedure are described in Algorithm 1.

It is worth noting that the initial mean $\mu_{1}$ and principal directions $\Gamma_{1}$ can be set simply by a zero vector $\mathbf{0}$ and $\delta \mathbf{I}$ with a small value $\delta$, respectively. Furthermore, a pair of appropriate initial ones can also be used for accelerating the convergence while a prior knowledge is given. For generating the kernel basis, the usual way is using the whole original data $X$ to generate the kernel basis, and obtain a full kernel. However, calculating a full kernel might cause the curse of high dimensionality and increase the computational cost. A reduced kernel can be used to replace the full kernel to avoid these problems $[11,12]$. The idea of reduced kernel is extracting $A$ from a small portion of $X$ for fast approximation. Also note that $A$ can be selected independently from $X$. This method is useful for large data problems, when $n$ goes to infinity.

\subsection{Asymptotic Stability}

When we gather a small number of observations, we can use batch KPCA to analyze them so that we can ensure that there are no outliers. Then, we can choose basis of kernel based on this small group. we show that in the iterative rKPCA $\mu_{t}$ converges to

$$
\frac{\mathrm{E}_{G}\left[\dot{\Psi}\left(z\left(h_{x}, \mu, \Gamma\right)\right) h_{x}\right]}{\mathrm{E}_{G}\left[\dot{\Psi}\left(z\left(h_{x}, \mu, \Gamma\right)\right)\right]},
$$

and $\Gamma_{t}$ converges to $\Gamma$ whose columns are the first $l$ eigenfunctions of

$$
\frac{\mathrm{E}_{G}\left[\dot{\Psi}\left(z\left(h_{x}, \mu, \Gamma\right)\right)\left(h_{x}-\mu\right) \otimes\left(h_{x}-\mu\right)\right]}{\mathrm{E}_{G}\left[\dot{\Psi}\left(z\left(h_{x}, \mu, \Gamma\right)\right)\right]}
$$

in descending eigenvalue order, respectively. That is, we can guarantee that the solution of our iterative rKPCA will converge to the one of the original rKPCA as shown in (8) and (9). Given the kernel basis at observations, if the initial values of $\mu$ and $\Gamma$ are close to stable points, $\mu_{t}$ and $\Gamma_{t}$ are asymptotically stable $[13,10]$. If outliers are the first data in our algorithm, then our method may lead to principal components which are very different from batch KPCA. To ensure the asymptotically stability. Asymptotic stability of $\mu$ is proved by the solution of $\lim _{t \rightarrow \infty} g_{t}^{(\mu)}$. Now we state the convergence properties of the online rKPCA in Theorems 1 and 2 (we put the proofs in Appendix). The theorem in [10] is applied to prove the asymptotic stability of $\Gamma$.

Theorem 1 (Asymptotic Stability of $\mu_{t}$ ). In our proposed iterative rKPCA, $\mu_{t}$ converges to $\frac{E_{G}\left[\dot{\Psi}\left(z\left(h_{x}, \mu, \Gamma\right)\right) h_{x}\right]}{E_{G}\left[\dot{\Psi}\left(z\left(h_{x}, \mu, \Gamma\right)\right)\right]}$ almost surely.

Theorem 2 (Asymptotic Stability of $\Gamma_{t}$ ). Assume that the leading $l$ eigenvalues of $\frac{E_{G}\left[\dot{\Psi}\left(z\left(h_{x}, \mu, \Gamma\right)\right)\left(h_{x}-\mu\right) \otimes\left(h_{x}-\mu\right)\right]}{\left.E_{G} \dot{\Psi}\left(z\left(h_{x} \mu, \Gamma\right)\right)\right]}$ have unit multiplicity. Given a finite kernel basis set $\left\{K\left(\cdot, u_{t^{\prime}}\right)\right\}_{t^{\prime}=1}^{\tilde{m}}$, when the initial $\Gamma_{0}$ is randomly assigned and the learning rate $\eta_{t}=\frac{1}{t}, \Gamma_{t}$ in the iterative $r K P C A$ converges to the matrix with columns of these l eigenvectors almost surely. Moreover, the updating system is locally asymptotically stable. 


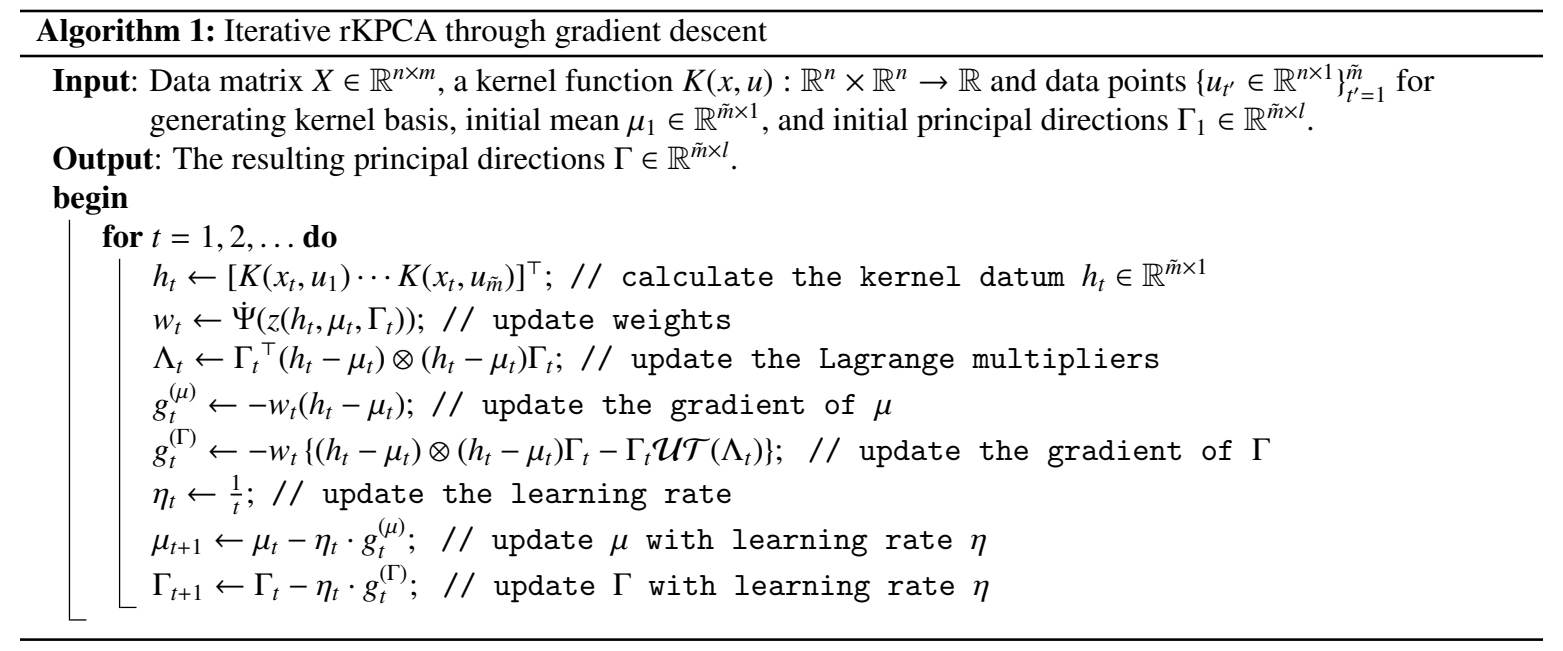

We use Ljung's theorem [13] to prove that the updating systems for the mean and principal components are asymptotically stable. We should notice that the asymptotically stable values of $\mu$ and $\Gamma$ are not equal to corresponding values resulted from batch KPCA of unpolluted data, but they are functions of the $\Psi$ function that contains outliers. Though the mean and principal components resulted from the iterative rKPCA do not converge to those from the KPCA of the unpolluted data, since the $\Psi$ assigns smaller weights on outliers, the mean and principal components are more closer to those from KPCA of the unpolluted data. Therefore they may lead to better data reconstruction than those resulted iterative KPCA directly. Another difficulty for the iterative rKPCA is that we cannot identify what are outliers when we only gather a few observations. Hence, if we collect outliers in the beginning of the iterative updating, the weight function will give lower weight on those really important data and thus lead to a biased results. Therefor. we propose a remedy approach is to obtain initial mean and principal components from batch KPCA when gathering a group of data in the beginning, then we start to do the iterative rKPCA. Moreover, we are supposed to know types of observations, so we can determine whether we can import data by random sampling or sequential neighbors.

\section{Experimental Results}

The behavior of iterative rKPCA is illustrated in four natural and synthetic experiments. The first example is a 2-dimensional synthetic example with polynomial kernel. The second one is the phoneme data, a func-
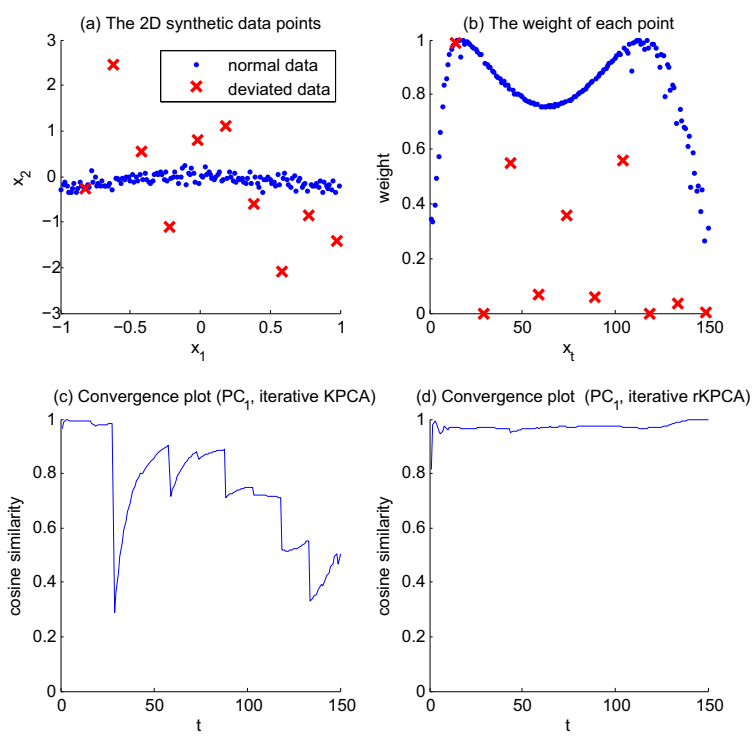

Figure 1: The convergence plots and the psi-principle weights of the iterative KPCA and rKPCA.

tional data, that has three principal components as a result of the previous work [6]. In the third and fourth examples, we use wavelet kernels for multiresolution analysis [14, 15]. Level-1 Haar wavelet kernel and Order-4 Symlet wavelet kernel [16] are applied to the Lena picture and a series of face images respectively in order to show how the iterative rKPCA reduces the influence of outliers in image reconstruction. In our experiments, we insert artificial contaminated data into the data as outliers. These results show that our 
proposed iterative rKPCA can extract the principal components close to those resulted from the data without outliers.

Example 1 (2-dimensional synthetic data) We generate this 2-dimensional data points $\left\{\left(x_{11}, x_{12}\right), \ldots,\left(x_{n 1}, x_{n 2}\right)\right\}$ with $n=150$, where $x_{i 1}$ 's are equally spaced over $(-1,1)$ and $x_{i 2}=-0.3 x_{i 1}^{2}+0.1 \mathrm{e}_{i}$, where $\mathrm{e}_{i}$ 's are iid from standard normal. For generating contaminated data, 10 points from the original data are replaced by points via $N\left( \pm 0.5, \sigma^{2}\right)$ in $x_{2}$ with $\sigma=1.5$ (five points for each). All these data points are plotted in Figure 1(a). Kernel data are made by using polynomial kernel $K(x, u)=\left(x^{\top} u\right)^{2}, x, u \in \mathbb{R}^{2}$.

To check the convergence of the principal directions, we use the absolute value of cosine similarity to measure the goodness of the estimated leading principal direction. Note that the absolute value of cosine similarity is defined as follows

$$
\text { cosine similarity }=\left|\frac{\langle\mathbf{v}, \mathbf{u}\rangle}{\|\mathbf{v} \mid\| \mathbf{u} \|}\right|,
$$

which is used for measuring the similarity between $\mathbf{v}$ and $\mathbf{u}$. A higher value represents that these two vectors are more identical. In our experiments, we apply batch KPCA with the non-contaminated data and use the solution as the true one (ie., v) for comparing those (ie., u) estimated from iterative KPCA and ours. In this example, we placed these 10 contaminated data points (replaced ten original data points) equally over the online updating procedure to observe the influence of the contaminated data more clearly. From Figure 1(c), it shows that the iterative KPCA can not resist the influence from the contaminated data. The estimated principal direction is affected by these 10 contaminated data and can not achieve to estimate the true principal direction. The absolute value of the cosine similarity by using the iterative KPCA is 0.5041 . On the contrary, our iterative rKPCA can reduce the influence of the contaminated data and reach good estimation of the true principal direction with the 0.9878 absolute value of the cosine similarity (see Figure 1(d)). Besides, the difference of the leading principal direction between rKPCA and iterative rKPCA in terms of the RKHS norm is 0.0296 . Figure 1(b) also shows that our iterative rKPCA decreases the weights of the contaminated data via the $\Psi_{1}$ function. Here we use $\beta=0.07$ in this 2-dimensional synthetic data. Based on these results, we evidence that our proposed method is more stable against outliers and can conclude a very similar solution to the true one. This also shows that our iterative rKPCA indeed converges to the solution of the original

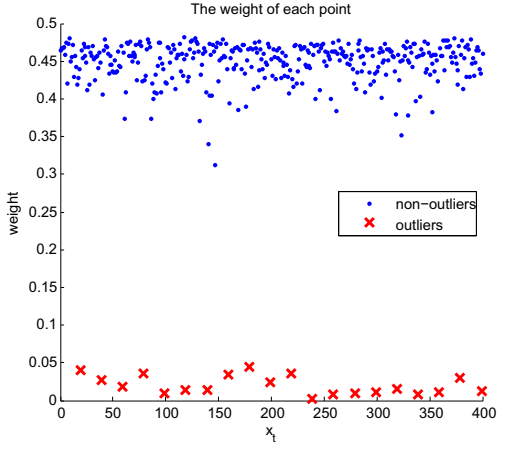

Figure 3: The weight of each functional datum.
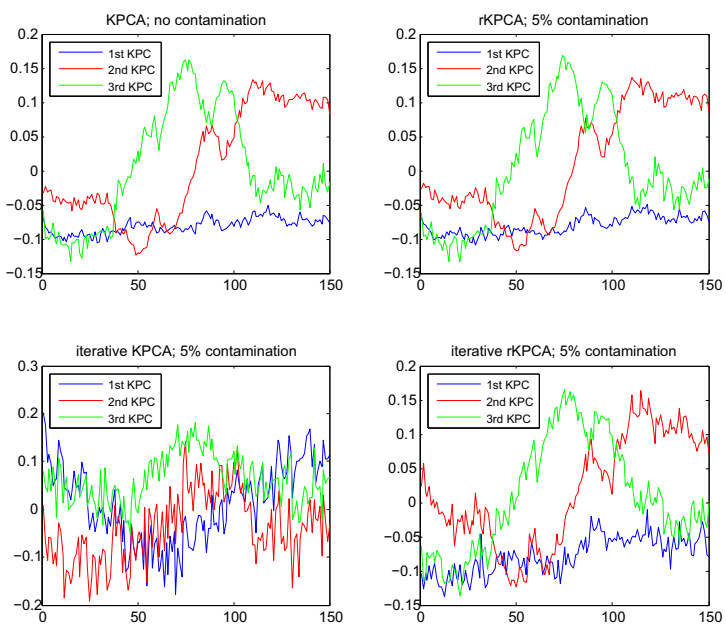

Figure 4: The three leading principal curves estimated by batch KPCA (no contamination), batch rKPCA (5\% contamination), iterative KPCA ( $5 \%$ contamination), and iterative rKPCA (5\% contamination.)

rKPCA.

Example 2 (Phoneme functional data) In this example, we evaluate our method with real world functional data. The functional phoneme data are extracted from the TIMIT database (TIMIT Acoustic-Phonetic Continuous Speech Corpus, NTIS, US Dept of Commerce $)^{1}$. Five phonemes for classification based on digitized speech are: "sh", "iy", "dcl", "aa", and "ao". This functional data consist of 2000 pairs of $\left\{\left(f_{i}, y_{i}\right)\right\}_{i=1}^{2000}$, where $y_{i}$ represents the class (phoneme) membership and $f_{i}$ is the discretized log-periodogram of length 150

\footnotetext{
${ }^{1}$ This dataset is available in http://www.lsp.upstlse.fr/staph/npfda/.
} 

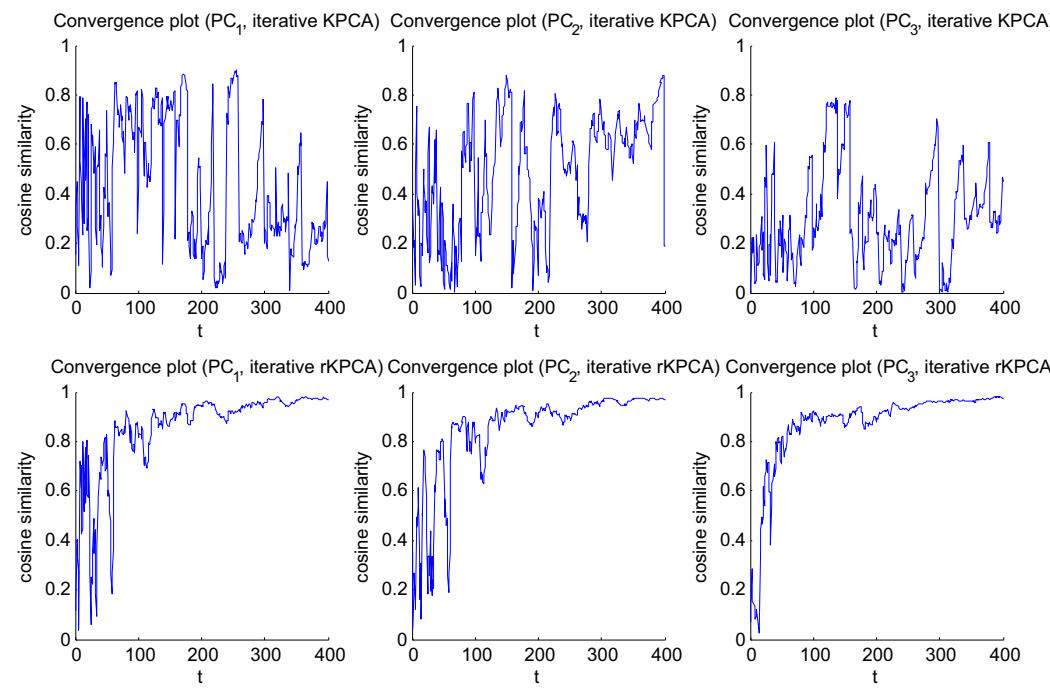

Figure 2: The convergence plots of the three leading principal components by the iterative KPCA and the iterative rKPCA.

frequencies. Our experiments focus on the iterative rKPCA, so only one group, "sh" sound with 400 curves are applied in our analysis. 5\% of data (20 curves) are randomly drawn to be contaminated with additive perturbation as $\tilde{f}_{i}(t)=f_{i}(t)+5 \cos (2 \pi t)+3 \mathrm{e}_{i}(t)$ with $t=0.5 / 150,1.5 / 150,2.5 / 150, \ldots 149.5 / 150$, where $e_{i}(t)$ are iid from uniform $(0,1)$. In this experiment, three leading functional principal directions are extracted from the contaminated data from the iterative KPCA and our iterative rKPCA. Note that we use the $\Psi_{2}$-based iterative rKPCA in this functional data example. The pair of parameters $(\beta, \eta)$ in $\Psi_{2}$ function is $(0.002,2)$.

For comparison of the performance of the estimated principal directions, we apply iterative KPCA, iterative rKPCA, and rKPCA to the contaminated data as the comparison. Note that we also apply KPCA to noncontaminated data for computing the true solution. Using the setting as Example 1, we placed these 5\% contaminated data points equally over the online updating procedure. In Figure 2, 3, and 4, we can find that our iterative rKPCA still can reach the true principal directions closely while the iterative KPCA is affected by the deviated data $\tilde{f}(t)$ and chooses wrong directions of principal components. We also can find the difference of the original rKPCA and iterative rKPCA is not significant where the difference in terms of the RKHS norm is 0.1256 . This also evidences that our iterative rKPCA can perform as well as the original one. We also report the absolute values of the cosine similarity between the

\begin{tabular}{|l|c|c|c|}
\hline & $P C_{1}$ & $P C_{2}$ & $P C_{3}$ \\
\hline iterative rKPCA & 0.9699 & 0.9677 & 0.9752 \\
\hline iterative KPCA & 0.1267 & 0.1909 & 0.4468 \\
\hline
\end{tabular}

Table 1: The absolute values of the cosine similarity between the true first three principal directions and the estimated principal directions by the iterative KPCA and the iterative rKPCA.

true first three principal directions and those generated by iterative KPCA and ours (See Table 1). Furthermore, we can observe that the psi-principle weights of the normal data are closer to each other than those generated by the $\Psi_{1}$ function.

Example 3 (Lena image) In this example, we test our method on the "Lena" image. Under compression ratio $1 / 8$, the iterative robust kernel principal component algorithm is evaluated by examining its use for image compression with level-1 Haar wavelet kernel. This image is contaminated by setting several $40 \times 40$ white blocks (see Figure 5(b)). It is a 256-gray-level image of size $512 \times 512$. The image was coded $8 \times 8$ disjoint blocks. Each block is arranged into a $64 \times 1$ column vector, so the image is a form of $64 \times 4096$. To reconstruct the image from eight principal components, the uncontaminated images are projected onto the subspace spanned by two basis sets from the iterative KPCA and iterative rKPCA respectively.

For the iterative KPCA, the estimated basis set is disarranged by the white block and could not converge 

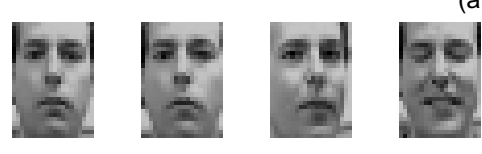

(a) Original images

(b) Fortune cat substitute

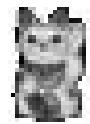

(c) Iterative wavelet KPCA
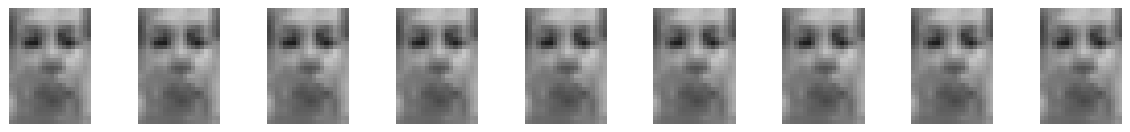

(d) Iterative wavelet rKPCA
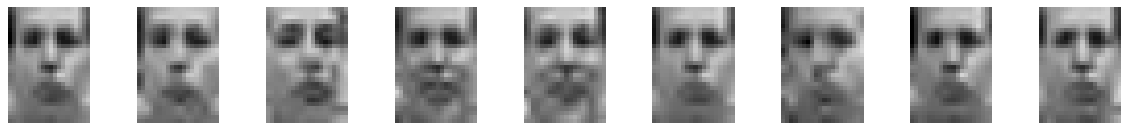

(e) Wavelet rKPCA
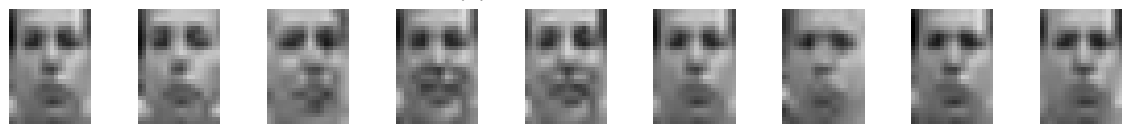

Figure 6: The original images are in the first row. The fortune cats which replace the images are in the second row. The reconstructed images resulted from the iterative KPCA are in the third row. The reconstructed images resulted from the iterative rKPCA are in the fourth row.

to the correct basis set in this one-pass updating procedure (see Figure 5(c)). This unstability is caused by the single datum updating procedure. The influence of outliers is enlarged while only using local information (single datum) to adjust the principal directions. On the other hand, Figure 5(d) shows that our iterative rKPCA with $\Psi_{1}$ function can resist the contamination of the white blocks and gives a better result of extracting the principal directions. Compared to the rKPCA, the iterative rKPCA also can perform as well as rKPCA in this case (see Figure 5(b)). In this image example, we use the parameter $\beta=3 \times 10^{-6}$ in $\Psi_{1}$ function and an initial matrix of $16 \times 8$ dimension where each entry is generated from $N(250,1)$.

Example 4 (Series images) In this series images example, there are 90 gray-level series human faces images with size $28 \times 20^{2}$. From 10th image to 90th image, every 10th image is replaced by the "fortune cat" image. The original images of the training set

\footnotetext{
${ }^{2}$ These series images data is available in http://www.cs.toronto.edu/ roweis/data.html
}

shown in Figure 7(a) are replaced by the Fortune Cats shown in Figure 7(b). Here the iterative KPCA and the iterative rKPCA uses the 81 human faces and 9 fortune cats as the training set and Order-4 symlet wavelet kernel. We apply the sym4 wavelet coefficients to extract the main resolution of each image, and use PCA to capture 9 principal components of these coefficients. The reconstructed images made by the iterative KPCA shown in Figure 7(c). The shadow of Fortune cats appear in those images reconstructed by the iterative KPCA. On the contrary, the iterative rKCA and rKPCA preserve the rotation of faces and leads to a cleared reconstructed images (see Figure 7(d) and 7(e)).

Example 5 (KDD Intrusion Detection Data) In this example, we consider the KDD intrusion detection dataset, which is usually used in large-scale problems. We use binary and continuous features ( 38 features) and focus on the training subset under the tcp protocol. We extract 60000 normal data points and thus the kernel matrix will be too large to be stored in practices. In this example, we replace $5 \%$ data from normal data by the denial-of-service attacks, which can be regarded as the 

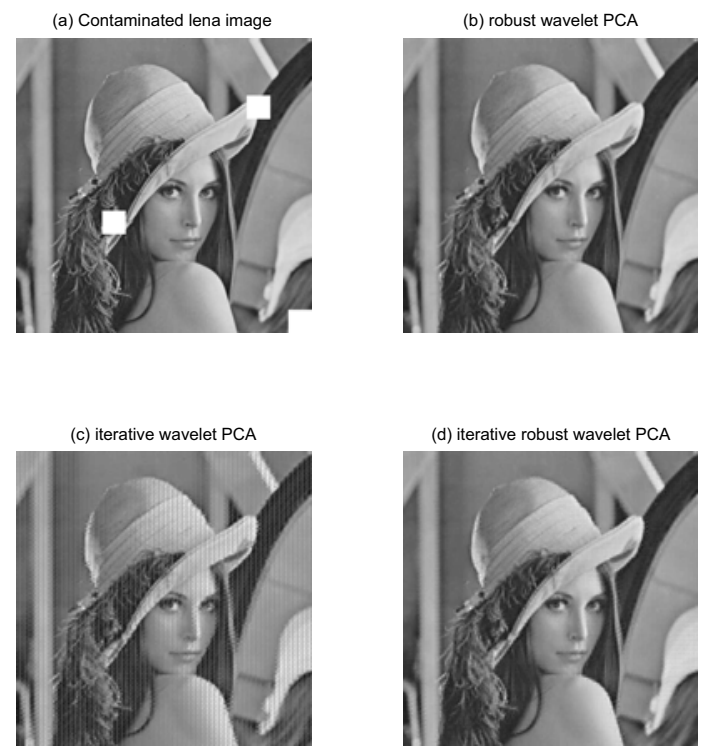

Figure 5: (a) original Lena image (b) image contaminated by three $40 \times 40$ pixels white windows (c) image recovered by the iterative conventional KPCA (d) image recovered by the iterative robust KPCA

outliers. The kernel data are also made by using polynomial kernel $K(x, u)=\left(x^{\top} u\right)^{2}, x, u \in \mathbb{R}^{2}$ as in Example 1 . Different to others examples, we cannot directly apply batch KPCA because of the data size in this case. Thus, we apply the iterative KPCA with non-contaminated and use the resulting solution as the true one. We also compared the cosine similarity between the true solution and the one estimated by iterative KPCA or ours. The resulting cosine similarities for iterative KPCA and ours are 0.0012 and 0.9811 respectively. As the same results with those in the previous examples, the bad performance shows that the iterative KPCA is affected by the attacks while ours is more robust against outliers.

\section{Conclusion}

We have presented a method for online robust kernel principal component analysis that can be used for nonlinear models from data that may be contaminated by outliers. The approach extends previous work in the image reconstruction by modeling outliers occur sparsely. The method has been applied on natural and synthetic data and shown improved robustness to outliers when compared with other techniques.

It is known that the KPCA is sensitive to outliers. There are various robustness techniques in a variety of ways. We work on applications of robustness-principle for rKPCA through automatic and iterative data weighting scheme. The mean and the principal components generated by the iterative rKPCA are asymptotically stable. Furthermore, we demonstrate the characteristics by experiments of synthetic and real data that the proposed method uses less time to calculate and have equivalent robustness as the batch KPCA.

Yet, how to tune the parameter of the psi-function still needs to be surveyed. Since we obtain the data sequentially, it is not reasonable to use the cross validation in tuning the parameters. Besides, the definition of outliers is challenging if we have only small number of initial data. Practically, it is better to provide appropriate initial principal components based on a small batch KPCA. Though these natural drawbacks exist in iterative KPCA. The robust weight function improves the robustness in high-dimensional data.

\section{Appendix: Proofs of Theorems 1 and 2}

\subsection{Prooffor Theorem 1}

Owning to the fact that the noises wash out [13], as $t$ is sufficiently large, we have an ordinary differential equation (ODE)

$$
\frac{d}{d t} \mu_{t}:=m\left(\Gamma_{t}, \mu_{t}\right)-\kappa\left(\Gamma_{t}, \mu_{t}\right) \mu_{t}
$$

where $m(t)=m\left(\Gamma_{t}, \mu_{t}\right)=\mathrm{E}_{G}\left[\dot{\Psi}\left(z\left(h_{x}, \mu_{t}, \Gamma_{t}\right)\right) h_{x}\right]$ and $\kappa(t)=\kappa\left(\Gamma_{t}, \mu_{t}\right)=\mathrm{E}_{G}\left[\dot{\Psi}\left(z\left(h_{x}, \mu, \Gamma\right)\right)\right]$. The ODE can be solved by using an integrating factor

$$
f(t)=\exp \left\{\int_{0}^{t} \kappa(s) d s\right\}
$$

on both sides, then we have

$$
\mu_{t}=\frac{\int_{0}^{t} m(s) f(s) d s+a}{f(t)}
$$

where $a$ is an arbitrary constant. Because $f^{\prime}(t)=$ $\kappa(t) f(t)>0$, this implies that $f(t) \rightarrow \infty$ as $t \rightarrow \infty$. By l'Hôpital's rule, $\mu_{t}$ converges to

$$
\frac{m(\infty)}{\kappa(\infty)}=\frac{\mathrm{E}_{G}\left[\dot{\Psi}\left(z\left(h_{x}, \mu, \Gamma\right)\right) h_{x}\right]}{\mathrm{E}_{G}\left[\dot{\Psi}\left(z\left(h_{x}, \mu, \Gamma\right)\right)\right]}
$$

as $t \rightarrow \infty$. 


\subsection{Prooffor Theorem 2}

There are six sufficient conditions for Theorem 2 in order that the principal components are asymptotically stable:

1. $\eta_{t}$ is a sequence of positive real numbers such that

$$
\eta_{t} \rightarrow 0, \sum_{t=1}^{\infty} \eta_{t}^{p}<\infty
$$

for $p>1$, and $\sum_{t=1}^{\infty} \eta_{t}=\infty$.

2. $\Gamma_{t}$ is bounded almost surely.

3. $g_{t}^{(\Gamma)}\left(\Gamma_{t}, h_{t}-\mu_{t}\right)$ is continuously differentiable in $\Gamma_{t}$ and $h_{t}-\mu_{t}$, and its derivative is bounded in time.

4. $\lim _{t \rightarrow \infty} E_{G}\left[g_{t}^{(\Gamma)}\left(\Gamma_{t}, h_{t}-\mu_{t}\right)\right]$ exists for $\Gamma_{t}$ in attraction $D(S)$.

5. There is a locally asymptotically stable set $S$ for the differential equation

$$
\frac{d}{d t} \Gamma_{t}=g_{t}^{(\Gamma)}
$$

with domain of attraction $D(S)$.

6. $\Gamma_{t}$ enters some compact subset $A \subset D(S)$ infinitely often with probability 1 .

Checking Conditions 1 to 4 Here we choose $\eta_{t}=\frac{1}{t}$ so that $\eta_{t}$ satisfies Condition 1. For Condition 2, $h_{t}$ is bounded by given a bounded basis set $\left\{K\left(\cdot, u_{t^{\prime}}\right)\right\}_{t^{\prime}=1}^{\tilde{m}}$ and a bounded input data $x_{t}$. For Condition 3, since $g^{(\Gamma)}$ is composed of differentiable functions $\dot{\Psi}, \Sigma \Gamma$ and $\Gamma \mathcal{U T}\left(\Gamma^{\top} \Sigma \Gamma\right)$, thus $g^{(\Gamma)}$ is continuously differentiable with respect to $\Gamma$. For the bounded derivative requirement, we have to show

$$
\sup _{h_{t}-\mu_{t}, \Gamma_{t}}\left|\frac{d}{d t} g_{t}^{(\Gamma)}\right|<\text { a constant. }
$$

Under a fixed kernel basis set, $\left|\dot{\Psi}_{t}\right| \leq 1$, bounded $\Gamma_{t}$ and Condition 2, $\left\|h_{t}-\mu_{t}\right\|$ is bounded. Since $\sup _{h_{t}-\mu_{t}, \Gamma_{t}}\left|\frac{d}{d t} g_{t}^{(\Gamma)}\right|$ is a tensor product of $\left|\dot{\Psi}_{t}\right|,\left\|h_{t}-\mu_{t}\right\|$ and $\Gamma_{t}$, thus $\sup _{h_{t}-\mu_{t}, \Gamma_{t}}\left|\frac{d}{d t} g_{t}^{(\Gamma)}\right|$ is bounded so that Condition 3 is satisfied. If $\lim _{t \rightarrow \infty} \mu_{t}$ and $\lim _{t \rightarrow \infty} \Gamma_{t}$ exist, then Condition 4 holds with $\lim _{t \rightarrow \infty} E_{G}\left[g_{t}^{(\Gamma)}\left(\Gamma_{t}, h_{t}-\mu\right)\right]=$ $E_{G}\left[\dot{\Psi}\left(z\left(h_{x}, \mu, \Gamma\right)\right)\left\{\left(h_{x}-\mu\right) \otimes\left(h_{x}-\mu\right) \Gamma-\Gamma \mathcal{U} \mathcal{T}(\Lambda)\right\}\right]$.

Since Theorem 1 confirms the existence of the limit of $\mu_{t}$, the rest part that we have to show is the existence of $\Gamma_{t}$. We show it in the procedure of checking Condition 5 .

Checking Condition 5 In the following analysis, we use ODE method to check Condition 5. The ODE derived from the expectation of $g_{t}^{(\Gamma)}\left(\Gamma_{t}, h_{t}-\mu\right)$ is as

$$
\frac{d \Gamma_{t}}{d t}=\Sigma^{\prime} \Gamma_{t}-\Gamma_{t} \mathcal{U} \mathcal{T}\left(\Gamma_{t}^{\top} \Sigma^{\prime} \Gamma_{t}\right)
$$

where $\frac{\mathrm{E}_{G}\left[\dot{\Psi}\left(z\left(h_{x}, \mu, \Gamma\right)\right)\left(h_{x}-\mu\right) \otimes\left(h_{x}-\mu\right)\right]}{\mathrm{E}_{G}\left[\dot{\Psi}\left(z\left(h_{x}, \mu, \Gamma\right)\right)\right]}=\Sigma^{\prime}$. Here we assume that all the eigenvalues of $\Sigma^{\prime}$ are not zero. This local asymptotic stability can be proved by Lyapunov's second method ([17]) and mathematical induction. We put the basis (base case) in Step 1 and the inductive step in Steps 2.

Step 1: Here we show that the solution of the ODE of the first eigenvector $\gamma_{1}(t)$ is locally asymptotically stable. We use the ODE method to show the convergence of $\gamma_{1}(t)$ and asymptotic stability by the differential equation

$$
\frac{d \gamma_{1}(t)}{d t}=\Sigma^{\prime} \gamma_{1}(t)-\gamma_{1}(t)\left(\gamma_{1}(t)^{\top} \Sigma^{\prime} \gamma_{1}(t)\right)
$$

Assume we choose $l$ principal components which are denoted as $q_{1}(t), q_{2}(t), \cdots, q_{l}(t)$ corresponding to eigenvalues $\lambda_{1}>\lambda_{2}>\cdots>\lambda_{l}$, then we can expand $\gamma_{1}(t)$ in terms of the entire orthonormal set of eigenvectors as

$$
\gamma_{1}(t)=\sum_{k=1}^{l} \theta_{k}(t) q_{k}, \text { where } \theta_{k}(t)=\gamma_{i}(t)^{\top} q_{k} .
$$

Plugging (33) together with $\Sigma^{\prime} q_{k}=\lambda_{k}(t) q_{k}$ into (32) gives

$$
\begin{aligned}
& \frac{d \gamma_{1}(t)}{d t}=\sum_{k=1}^{l} \frac{d \theta_{k}(t)}{d t} q_{k} \\
= & \sum_{k \geq i} \lambda_{k}(t) \theta_{k}(t) q_{k}-\left(\sum_{v=1}^{l} \lambda_{v}(t) \theta_{v}^{2}(t)\right)\left(\sum_{k=1}^{l} \theta_{k}(t) q_{k}\right) \\
= & \sum_{k \geq 1}\left(\lambda_{k}(t)-\sum_{l=1}^{l} \lambda_{l}(t) \theta_{l}^{2}(t)\right) \theta_{k}(t) q_{k}:=\sum_{k=1}^{l} \frac{d \theta_{k}(t)}{d t} q_{k},
\end{aligned}
$$

where the last equality results from the orthonormality of eigenvectors. Since $\gamma_{1}(t)=\sum_{v=1}^{l} \theta_{v}(t) q_{v}$ and $\Sigma^{\prime}\left(\sum_{v=1}^{l} \theta_{v}(t) q_{v}\right)=\sum_{v=1}^{l} \lambda_{v} \theta_{v} q_{v}$, hence the second term of (32) can be written as

$$
\begin{aligned}
& \left(\gamma_{1}(t)^{\top} \Sigma^{\prime} \gamma_{1}(t)\right) \gamma_{1}(t) \\
= & {\left[\left(\sum_{u=1}^{l} \theta_{u} q_{u}\right)^{\top} \sum_{v=1}^{l} \lambda_{v} \theta_{v} q_{v}\right]\left[\sum_{k=1}^{l} \theta_{k} q_{k}\right] } \\
= & \left(\sum_{v=1}^{l} \lambda_{v} \theta_{v}^{2}\right)\left(\sum_{k=1}^{l} \theta_{k} q_{k}\right),
\end{aligned}
$$

where the last equality results from the orthonormality of eigenvectors. Then we can multiply $q_{k}^{\top}$ to both sides of (34), and therefore by the orthonormality of $\left\{q_{k}\right\}_{k=1}^{l}$

$$
\frac{d \theta_{k}(t)}{d t}=\theta_{k}(t)\left(\lambda_{k}(t)-\sum_{i=1}^{l} \lambda_{i}(t) \theta_{i}^{2}(t)\right) .
$$


As $t \rightarrow \infty,\left\{\lambda_{v}\right\}_{v=1}^{l}$ are independent of $t$. Thus we have the asymptotic ODE

$$
\frac{d \theta_{k}(t)}{d t}=\theta_{k}(t)\left[\lambda_{k}-\lambda_{1} \theta_{1}^{2}(t)-\sum_{v=2}^{l} \lambda_{v}^{2} \theta_{v}^{2}(t)\right] .
$$

Then the solution of the ODE depends on the number of the basis of $\Sigma^{\prime}, l$, which is either greater than one or equal to one.

Case $l>1$ : When $l \geq 2$, as $t \rightarrow \infty\left\{\theta_{k}(t)\right\}_{k \geq 2}$ is not empty. Then we can define a variable $\omega_{k}(t)$ by $\theta_{k}(t) / \theta_{i}(t)$ (here $\theta_{i} \neq 0$ ), and (37) implies the following ODE

$\frac{d \omega_{k}(t)}{d t}=\frac{1}{\theta_{i}(t)}\left(\frac{d \theta_{k}(t)}{d t}-\omega_{k} \frac{d \theta_{i}(t)}{d t}\right)$ for all $k=1, \ldots, l$.

After replacing $\frac{d \theta_{k}(t)}{d t}$ and $\frac{\theta_{i}(t)}{d t}$ by the right hand side of (37), we have

$$
\begin{aligned}
\frac{d \omega_{k}(t)}{d t}= & \frac{1}{\theta_{i}(t)}\left[\theta_{k}(t)\left(\lambda_{k}-\sum_{k=1}^{l} \lambda_{k} \theta_{k}^{2}(t)\right)\right. \\
& \left.-\omega_{k} \theta_{i}(t)\left(\lambda_{i}-\sum_{k=1}^{l} \lambda_{k} \theta_{k}^{2}(t)\right)\right]
\end{aligned}
$$

which can be simplified to

$$
\frac{d \omega_{k}(t)}{d t}=\omega_{k}(t)\left(\lambda_{k}-\lambda_{i}\right)
$$

The solution to the above differential equation is

$$
\omega_{k}(t)=\omega_{k}(0) \exp \left[\left(\lambda_{k}-\lambda_{i}\right) t\right] .
$$

Since the eigenvalues are indexed in decreasing order, $\lambda_{i}$ is the largest eigenvalue in $\left\{\lambda_{i}, \ldots, \lambda_{l}\right\}$, and $\lambda_{k}-\lambda_{i}<0$ for $k>i$. Therefore, $\omega_{k}(t)$ exponentially converges to zero with any $\omega_{k}(0)$ for $k>1$.

Case $l=1$ : By the equation (37),

$$
\begin{aligned}
\frac{d \theta_{1}(t)}{d t} & =\theta_{1}(t)\left[\lambda_{1}-\sum_{v=1}^{l} \lambda_{v} \theta_{1}^{2}(t)\right] \\
& =\theta_{1}(t)\left[\lambda_{1}-\lambda_{1} \theta_{1}^{2}(t)-\sum_{v=2}^{l} \lambda_{v} \theta_{v}^{2}(t)\right] .
\end{aligned}
$$

Since there is only one principal component, thus $\theta_{v}(t) \rightarrow 0$ for $v>1$ so that the last term above approaches zero. Therefore, we drop the last term, and (37) becomes

$$
\frac{d \theta_{1}(t)}{d t}=\lambda_{1} \theta_{1}(t)\left[1-\theta_{1}^{2}(t)\right] .
$$

To show that the solution of the above ODE is locally asymptotically stable, we need a Lyapunov function, $V(t)$, satisfying the following requirement:
1. $V(t)>(<) 0$,

2. $\frac{d}{d t} V(t)<(>) 0$

3. $V(t)$ has a minimum (maximum).

Define $V(t)=\left[\theta_{1}^{2}-1\right]^{2}$. Then we can use the Lyapunov theorem to show the asymptotic stability of $\gamma_{t}$. If there is a Lyapunov function for a $\frac{d}{d t} \theta_{1}(t)$, then the following differential solution is asymptotically stable:

$\frac{d V(t)}{d t}=\frac{d}{d t}\left[\theta_{1}^{2}-1\right]^{2}=4 \theta_{1}\left[\theta_{i}^{2}-1\right] \frac{d \theta_{1}}{d t}=-4 \lambda_{1} \theta_{1}^{2}\left[\theta_{1}^{2}-1\right]^{2}$.

Accordingly, $\frac{V(t)}{d t} \leq 0$. Thus $V(t)$ is a Lyapunov function and has a minimum at $\left|\theta_{1}\right|=1$ and $\theta_{1}=0$, which we have excluded by the assumption. Therefore, $\frac{V(t)}{d t}<0$, and then the system is locally asymptotically stable.

Step 2: By the updating of $\gamma_{k}$, we have the ODE

$$
\frac{d \gamma_{k}(t)}{d t}=\Sigma^{\prime} \gamma_{k}(t)-\gamma_{k}(t) \mathcal{U T}\left(\gamma_{k}(t)^{\top} \Sigma^{\prime} \gamma_{k}(t)\right)
$$

Expanding of each column of $\Sigma^{\prime}$ in terms of the eigenvectors of $\Sigma^{\prime}$ is

$$
\gamma_{k}(t)=\sum_{v=1}^{l} \theta_{v}(t) q_{v}
$$

and combining (46) and (45), we have

$$
\begin{aligned}
\frac{d \gamma_{k}(t)}{d t}= & \Sigma^{\prime} \gamma_{k}(t)-\left(\left(\gamma_{k}(t)\right)^{\top} \Sigma^{\prime} \gamma_{k}(t)\right) \gamma_{k}(t) \\
& -\sum_{v<k}\left(\left(\gamma_{k}(t)\right)^{\top} \Sigma^{\prime} \theta_{k} q_{k}\right) \theta_{v} q_{v} .
\end{aligned}
$$

Hence, the third term of (47) can be written as

$$
\begin{aligned}
& \sum_{v<k}\left(\left(\gamma_{k}(t)\right)^{\top} \Sigma^{\prime} \theta_{v} q_{v}\right) \theta_{v} q_{v} \\
= & \sum_{v<k}\left(\left(\gamma_{k}(t)\right)^{\top} \theta_{v} \Sigma^{\prime} q_{v}\right) q_{v} \\
= & \sum_{v<k} \lambda_{v}\left(\left(\gamma_{k}(t)\right)^{T} q_{v}\right) q_{v}=\sum_{v<k} \lambda_{v} \theta_{v}(t) q_{v}
\end{aligned}
$$

such that

$$
\begin{aligned}
& \Sigma^{\prime} \gamma_{k}(t)-\sum_{v<k}\left(\left(\gamma_{k}(t)\right)^{\top} \Sigma^{\prime} \theta_{v} q_{v}\right) \theta_{v} q_{v} \\
= & \sum_{v=1}^{l} \lambda_{v} \theta_{v}(t) q_{v}-\sum_{v<k} \lambda_{v} \theta_{v}(t) q_{v} \\
= & \sum_{v \geq k} \lambda_{v} \theta_{v}(t) q_{v} .
\end{aligned}
$$

As $t$ is large enough, $\left\{\lambda_{v}\right\}_{v=1}^{l}$ are independent of $t$. After plugging the above expansion together with $\Sigma^{\prime} q_{k}=\lambda_{k} q_{k}$ 
into (47), we have

$$
\begin{aligned}
\frac{d \gamma_{k}(t)}{d t}= & \sum_{u \geq k} \lambda_{v} \theta_{v}(t) q_{v}-\left(\sum_{u=1}^{l} \lambda_{u} \theta_{u}^{2}(t)\right)\left(\sum_{v=1}^{l} \theta_{v} q_{v}\right) \\
= & \sum_{u \geq k}\left(\lambda_{u}-\sum_{v=1}^{l} \lambda_{v} \theta_{v}^{2}(t)\right) \theta_{u}(t) q_{u} \\
& -\sum_{u<k}\left(\sum_{v=1}^{l} \lambda_{v} \theta_{v}^{2}(t)\right) \theta_{u}(t) q_{u} \\
:= & \sum_{u=1}^{l} \frac{d \theta_{u}(t)}{d t} q_{u},
\end{aligned}
$$

where

$$
\frac{d \theta_{u}(t)}{d t} \equiv \begin{cases}\theta_{u}(t)\left(\lambda_{u}-\sum_{v=1}^{l} \lambda_{v} \theta_{v}^{2}(t)\right), & \text { for } u \geq k \\ -\theta_{u}(t) \sum_{v=1}^{l} \lambda_{v} \theta_{v}^{2}(t), & \text { for } u<k\end{cases}
$$

For $u<k$, the solution to the differential equation is

$$
\theta_{u}(t)=\theta_{u}(0) \exp \left[-\left(\sum_{v=1}^{l} \lambda_{v} \theta_{v}^{2}\right) t\right.
$$

Moreover, since $\Sigma^{\prime}$ is positive definite, $\lambda_{v}>0$. Thus $-\left(\sum_{v=1}^{l} \lambda_{v} \theta_{v}^{2}\right)<0$, and then $\theta_{u}(t)$ converges to zero exponentially with any $\theta_{u}(0)$ for $k<j$.

When $u>k$, we can define a variable $\omega_{u}$ by $\theta_{u} / \theta_{k}$ (here $\theta_{k} \neq 0$ ), and then prove local asymptotic stability of solutions of the following ODE

$$
\frac{d \omega_{u}(t)}{d t}=\frac{1}{\theta_{k}(t)}\left(\frac{d \theta_{u}(t)}{d t}-\omega_{u} \frac{d \theta_{k}(t)}{d t}\right)
$$

After replacing $\frac{d \theta_{u}(t)}{d t}$ and $\frac{d \theta_{k}(t)}{d t}$ by the right hand side of (51), we have

$$
\begin{aligned}
\frac{d \omega_{u}(t)}{d t}= & \frac{1}{\theta_{k}}\left[\theta_{u}(t)\left(\lambda_{u}-\sum_{v=1}^{l} \theta_{v}^{2}(t) \lambda_{v}\right)\right. \\
& \left.-\omega_{u}(t) \theta_{k}(t)\left(\lambda_{k}-\sum_{v=1}^{l} \theta_{v}^{2} \lambda_{v}\right)\right],
\end{aligned}
$$

which is simplified to

$$
\frac{d \omega_{u}(t)}{d t}=\omega_{u}(t)\left(\lambda_{u}-\lambda_{k}\right) .
$$

The solution to the above differential equation is

$$
\omega_{u}(t)=\omega_{u}(0) \exp \left[\left(\lambda_{u}-\lambda_{k}\right) t\right]
$$

Since $\lambda_{u}<\lambda_{k}$, thus $\omega_{u}(t)$ converges to zero exponentially with an arbitrary $\omega_{u}(0)$ for all $u>k$.
When $u=k$, (51) implies that

$$
\frac{d \theta_{k}(t)}{d t}=\theta_{k}(t)\left(\lambda_{k}-\theta_{k}(t) \sum_{v>k} \lambda_{v} \theta_{v}^{2}(t)-\theta_{k}(t) \sum_{v<k} \lambda_{v} \theta_{v}^{2}(t)\right) \text {. }
$$

It has been shown in the previous cases that $\theta_{v}(t) \rightarrow 0$ for $v<k$ and $\theta_{v}(t) \rightarrow 0$ for $v>k$. Hence we can drop the last two terms, and the equation becomes

$$
\frac{d \theta_{k}(t)}{d t}=\theta_{k}(t) \lambda_{k}\left(1-\theta_{k}^{2}(t)\right)
$$

To show that $\theta_{j}(t)$ converges, we define another function

$$
P=\left(\theta_{k}^{2}(t)-1\right)^{2}
$$

After plugging (58) into $\frac{d P(t)}{d t}$, we have

$$
\frac{d P(t)}{d t}=-4 \lambda_{k} \theta_{k}^{2}(t)\left(\theta_{k}^{2}(t)-1\right)^{2}
$$

so we observe that $\frac{d P(t)}{d t} \leq 0$. Since $\theta_{k}^{2}(t) \neq 1$, thus $\frac{d P(t)}{d t}<0$, and hence the solution is locally asymptotically stable. Moreover, $\left\{\gamma_{k}(t)\right\}_{k=1}^{l}$ converge almost surely to $\pm q_{1}$, the normalized principal eigenvector of $\Sigma^{\prime}$.

Checking Condition 6 For Condition 6, the existence of the basin of attraction is needed to be checked. That is, there is a compact set $A$ of the set of all matrices such that $\Gamma_{t} \in A$ as $t \rightarrow \infty$. Here we show that there is a compact subset $D(S)$ of the set of all matrices such that $\Gamma_{t} \in D(S)$ as $t \rightarrow \infty$. Let $A$ be the compact subset of $\mathbb{R}^{\tilde{m} \times l}$ given by the set of matrices with norm less than or equal to some constant $b$. By the properties of an operator norm which is defined as $\|L\|:=\max _{x \neq 0} \frac{\|L x\|}{\|x\|}$,

$$
\begin{aligned}
\left\|\Gamma_{t+1}\right\| \leq & \left\|\eta_{t} \dot{\Psi}\left(h_{t}-\mu_{t}\right) y(t)\right\| \\
& +\left\|I-\dot{\Psi}_{t} \eta_{t} \mathcal{U} \mathcal{T}(\Lambda)\right\|\left\|\Gamma_{t}\right\| \\
\leq & \mid \eta_{t}\left\|\left(h_{t}-\mu_{t}\right)^{\top} y(t)\right\| \\
& +\left\|I-\dot{\Psi}_{t} \eta_{t} \mathcal{U} \mathcal{T}\left(y(t)^{\top} y(t)\right)\right\|\left\|\Gamma_{t}\right\|,
\end{aligned}
$$

where $y(t)=\left(h_{t}-\mu_{t}\right)^{\top} \Gamma_{t}$. Since $\|y(t)\|,\left\|h_{t}-\mu_{t}\right\|$ and $\left|\dot{\Psi}_{t}\right|$ are all bounded, and $\eta_{t} \rightarrow 0$ as $t \rightarrow \infty$, thus $\left|\dot{\Psi}_{t} \eta_{t}\right| \rightarrow 0$ so that $\left\|I-\dot{\Psi}_{t} \eta_{t} \mathcal{U} \mathcal{T}\left(y(t)^{\top} y(t)\right)\right\| \rightarrow 1$. This implies that $\left\|\Gamma_{t+1}\right\| \leq\left\|\Gamma_{t}\right\|$ when $t \rightarrow \infty$. Thus $\Gamma_{t}$ will be eventually in $A$ as $t \rightarrow \infty$. Since the basin of attraction $D(S)$ includes all matrices with bounded norm, thus $A$ belongs to $D(S)$. By the virtue of Ljung's theorem [13], the above checking ensures that $\Gamma_{t}$ converges to the matrix whose columns are the first $l$ eigenvectors of $\Sigma^{\prime}$ in descending eigenvalues order. 


\section{References}

[1] I. T. Jolliffe, Principal Component Analysis, Springer, 2002.

[2] B. Schölkopf, A. J. Smola, K.-R. Müller, Nonlinear component analysis as a kernel eigenvalue problem, Neural Computation 10 (5) (1998) 1299-1319.

[3] S. Günter, N. N. Schraudolph, S. V. N. Vishwanathan, Fast iterative kernel principal component analysis, Journal of Machine Learning Research 8 (2007) 1893-1918.

[4] J. E. Jackson, A User's Guide to Principal Components, Wiley and Sons, 1991.

[5] I. Higuchi, S. Eguchi, Robust principal component analysis with adaptive selection for tuning parameters, Journal of Machine Learning Research 5 (2004) 453-471.

[6] S.-Y. Huang, Y.-R. Yeh, S. Eguchi, Robust kernel principal component analysis, Neural Computation 21 (2009) 3179-3213.

[7] Y.-W. Pang, L. Wang, Y. Yuan, Generalized kpca by adaptive rules in feature space, International Journal of Computer Mathematics 87 (5) (2010) 956-968.

[8] L. Wang, Y.-W. Pang, D.-Y. Shen, N.-H. Yu, An iterative algorithm for robust kernel principal component analysis, in: Proceeding of International Conference on Machine Learning and Cybernetics, 2007.

[9] K. I. Kim, M. O. Franz, B. Schölkopf, Iterative kernel principal component analysis for image modeling, IEEE Transactions on Pattern Analysis and Machine Intelligence 27 (9) (2005) 13511366.

[10] T. D. Sanger, Optimal unsupervised learning in a single-layer linear feedforward neural network, Neural Networks 2 (6) (1989) 459-473.

[11] Y.-J. Lee, S.-Y. Huang, Reduced support vector machines: A statistical theory, IEEE Transactions on Neural Networks 18 (1) (2007) 1-13.

[12] Y.-J. Lee, O. L. Mangasarian, Rsvm: Reduced support vector machines, in: Proceedings of the First SIAM International Conference on Data Mining, 2001.

[13] L. Ljung, Analysis of recursive stochastic algorithms, IEEE Transactions on Automatic Control 22 (4) (1977) 551-575.

[14] S. Canu, X. Mary, A. Rakotomamonjy, Functional Learning Through Kernel, IOS Press, Amsterdam, 2003.

[15] B. Vidakovic, Statistical Modeling by Wavelets, Wiley Interscience, 1999.

[16] I. Daubechies, Ten Lectures on Wavelets, SIAM, Philadelphia, 1992

[17] E. Oja, A simplified neuron model as a principal component analyzer, Journal of Mathematical Biology 15 (3) (1982) 267 273. 